\title{
Neuroendoscopic stent placement for cerebrospinal fluid pathway obstructions in adults
}

\author{
Sascha Marx, MD, ${ }^{1}$ Steffen K. Fleck, MD, ${ }^{1}$ Ehab El Refaee, MD, ${ }^{1,2}$ Jotham Manwaring, MD, ${ }^{3}$ \\ Christina Vorbau, ${ }^{1}$ Michael J. Fritsch, MD, PhD, ${ }^{1}$ Michael R. Gaab, MD, PhD, ${ }^{4}$ \\ Henry W. S. Schroeder, MD, PhD, ${ }^{1}$ and Joerg Baldauf, MD, PhD'
}

\begin{abstract}
${ }^{1}$ Department of Neurosurgery, University Medicine Greifswald, Germany; ${ }^{2}$ Department of Neurosurgery, Kasr Alainy Research and Teaching Hospital, Cairo University, Egypt; ${ }^{3}$ Department of Neurosurgery and Brain Repair, University of South Florida, Tampa, Florida; and ${ }^{4}$ Hannover, Germany
\end{abstract}

\begin{abstract}
OBJECTIVE Since its revival in the early 1990s, neuroendoscopy has become an integral component of modern neurosurgery. Endoscopic stent placement for treatment of CSF pathway obstruction is a rarely used and underestimated procedure. The authors present the first series of neuroendoscopic intracranial stenting for CSF pathway obstruction in adults with associated results and complications spanning a long-term follow-up of 20 years.
\end{abstract}

METHODS The authors retrospectively reviewed a prospectively maintained clinical database for endoscopic stent placement performed in adults between 1993 and 2013.

RESULTS Of 526 endoscopic intraventricular procedures, stents were placed for treatment of CSF disorders in 25 cases (4.8\%). The technique was used in the management of arachnoid cysts (ACs; $n=8$ ), tumor-related CSF disorders $(n=13)$, and hydrocephalus due to stenosis of the foramen of Monro $(n=2)$ or aqueduct $(n=2)$. The mean follow-up was 87.1 months. No deaths or infections occurred that were related to endoscopic placement of intracranial stents. Late stent dislocation or migration was observed in 3 patients (12\%).

CONCLUSIONS Endoscopic intracranial stent placement in adults is rarely required but is a safe and helpful technique in select cases. It is indicated when reliable and long-lasting restoration of CSF pathway obstructions cannot be achieved with standard endoscopic techniques. In the treatment of tumor-related hydrocephalus, it is a good option to avoid reclosure of the restored CSF pathway by tumor growth. Currently, routine stent placement after endoscopic fenestration of ACs is not recommended. Stent placement for treatment of CSF disorders due to tumor is a good option for avoiding CSF shunting. To avoid stent migration and dislocation, and to allow for easy removal if needed, the device should be fixed to a bur hole reservoir.

http://thejns.org/doi/abs/10.3171/2015.7.JNS151005

KEY WORDS CSF disorders; intracranial stenting; neuroendoscopy; hydrocephalus

$\mathrm{S}$ INCE its revival in the early 1990s, neuroendoscopy has become an integral component of modern neurosurgery. ${ }^{7,16,19}$ Various endoscopic techniques have been described and are frequently used for the treatment of CSF circulation disorders including third ventriculostomy, septostomy, lamina terminalis fenestration, temporal ventriculostomy, foraminoplasty of the foramen of Monro, and aqueductoplasty. ${ }^{21}$ Endoscopic placement of indwelling stents, however, is a rarely performed, but nonetheless valuable, procedure. The best-studied stenting procedure is aqueductal stenting in the setting of triventricular hy- drocephalus and in cases of isolated fourth ventricles in children, which is known to be technically feasible and has shown good results in long-term follow-up., ${ }^{2,9,13,18,22}$ Limited reports exist, however, about the value and outcome of endoscopic stent placement in adults.

We present the first series of neuroendoscopic stent placement in adults from a single center over 20 years. The aim of the present study was to identify favorable indications for neuroendoscopic stent placement in adults, including operative results, complications, and clinical outcomes data. 


\section{Methods}

\section{Data Collection}

Since 1993, all neuroendoscopic procedures in the Department of Neurosurgery, University of Greifswald, have been gathered in a prospectively maintained clinical database. The authors retrospectively reviewed the database for intracranial stent placement in adults (age $\geq 18$ ) from 1993 to 2013. Data collection included diagnosis, operative procedure, associated complications, need for redo surgery, and clinical data from the perioperative period and follow-up. Subgroup analysis was performed based on diagnosis. End points were defined as the last documented follow-up or death. Patients lacking follow-up were excluded from follow-up analysis, but the reason for lack of follow-up was recorded. Complications were categorized as operative and nonoperative. Stent-related infection has been defined as ventriculitis or meningitis in the perioperative period or in follow-up, including increased CSF values of leukocytes, protein, lactate, and positive bacterial cultures. Clinical data were analyzed with a focus on change in symptoms (e.g., improvement of initial symptoms).

\section{Operative Technique}

All surgeries were performed with the patient under general anesthesia. The procedures between 1993 and 2006 were performed using the Gaab Universal Neuroendoscopic System (Karl Storz GmbH). Since 2007, every procedure has been performed using the "LOTTA" ventriculoscope (Karl Storz GmbH) ${ }^{20}$ The location of the entry point and the approach trajectory were selected according to the findings obtained from MRI or while using neuronavigation. A $2.5-\mathrm{cm}$ linear incision was made followed by placement of a $10-\mathrm{mm}$ bur hole. The dura was coagulated and opened to allow unimpeded passage of the endoscopic sheath. The endoscopic sheath with the trocar inside was inserted into the target ventricle or cyst with a "freehand" technique or under navigational guidance and secured in place with an endoscope-holding arm. Usually, the ventricle or cyst was then inspected using a $30^{\circ}$ and $45^{\circ}$ scope to gain a clear understanding of the anatomy. Thereafter, the primary component of the procedure was performed, i.e., tumor biopsy, cystocisternostomy, or endoscopic third ventriculostomy (ETV). The stent was created from a standard silicone ventricular or fimbria catheter (outer diameter $2.7 \mathrm{~mm}$ ). The latter contains small radial fimbria in the distal centimeter of the catheter, which provides increased anchoring. Additional perforations were made at certain distances along its length with Luer forceps or scissors to allow for communication between different CSF compartments. Perforations were not made where the catheter was to pass through brain parenchyma, the aqueduct, or the foramen of Monro, as a means of minimizing the risk of stent occlusion by glial tissue ingrowth. After preparation, the premeasured stent was placed through the main working channel of the ventriculoscope to the target depth under endoscopic visualization. The endoscopic sheath was then removed and the correct stent position checked with a small diagnostic scope passed along the stent through the cortical puncture channel. The placement of an aqueductal stent has been described previously. ${ }^{9}$ In brief, a prefrontal bur hole is used to get a valuable trajectory straight in front of the aqueduct. After cautious aqueductoplasty, a small diagnostic scope is introduced into the fourth ventricle. Hereafter, the stent is placed through the aqueduct into the fourth ventricle. In the last 8 years, the stents have been connected to a bur hole reservoir. Finally, the skin is closed in layers. Usually, the patients are observed overnight in the intermediate care unit.

\section{Results \\ Main Results}

During the study period, 526 endoscopic procedures were performed, 25 (4.8\%) of which involved placement of a stent. Twelve female and 13 male patients (mean age 43.2 years, range 18 to 70 years) were operated on. Endoscopic stent placement was used in the management of ACs $(\mathrm{n}=$ $8)$, obstructive hydrocephalus secondary to tumor $(n=13)$, and hydrocephalus due to stenosis of the foramen of Monro $(\mathrm{n}=2)$ and aqueduct $(\mathrm{n}=2)$. The mean follow-up duration for 19 of 25 patients was 87.1 months (range 1-239 months). Five patients from the tumor-related hydrocephalus group were lost to follow-up. One patient died 2 weeks after surgery due to respiratory failure. Endoscopic stent placement was the primary procedure in 8 patients and secondary in 17. Endoscopic procedures other than stent placement included septostomy, ETV, tumor biopsy, and partial tumor resection. The types of stents included both round $(n=17)$ and fimbria catheters $(n=8), 5$ of which were fixed to a bur hole reservoir. No stent-related infections occurred. Clinical symptoms immediately improved after the operation in all but 2 patients who suffered from persistent headache after stent placement for therapy of an AC. One stent was lost within the ventricular system during endoscopic intervention and could not be retrieved (Case 15). Late stent migration was observed in 1 patient $(5 \%)$ but required no redo surgery (Case 24). Late stent dislocation was observed in 2 patients (10\%) requiring 1 emergency (Case 2) and 1 elective (Case 25) redo surgery. Detailed information regarding patient characteristics, diagnosis, procedures, and follow-up is provided in Table 1. Information regarding patient selection and analysis is provided in Fig. 1.

\section{Stent Placement for Treatment of Arachnoid Cysts}

It is our practice to offer endoscopic fenestration, with or without stent placement, to adult patients with an AC and progressing clinical symptoms. Preoperative radiological evaluation has always involved the use of either fast imaging using steady-state acquisition or constructive interference in steady-state imaging since their development. These sequences help clarify CSF compartments and membrane anatomy. Endoscopic arachnoid cyst fenestration with cystocisternostomy and stent placement was performed in 8 patients with frontotemporal ACs. Headache, dizziness, and hemiparesis were the presenting clinical symptoms. Postoperative MRI showed a de- 


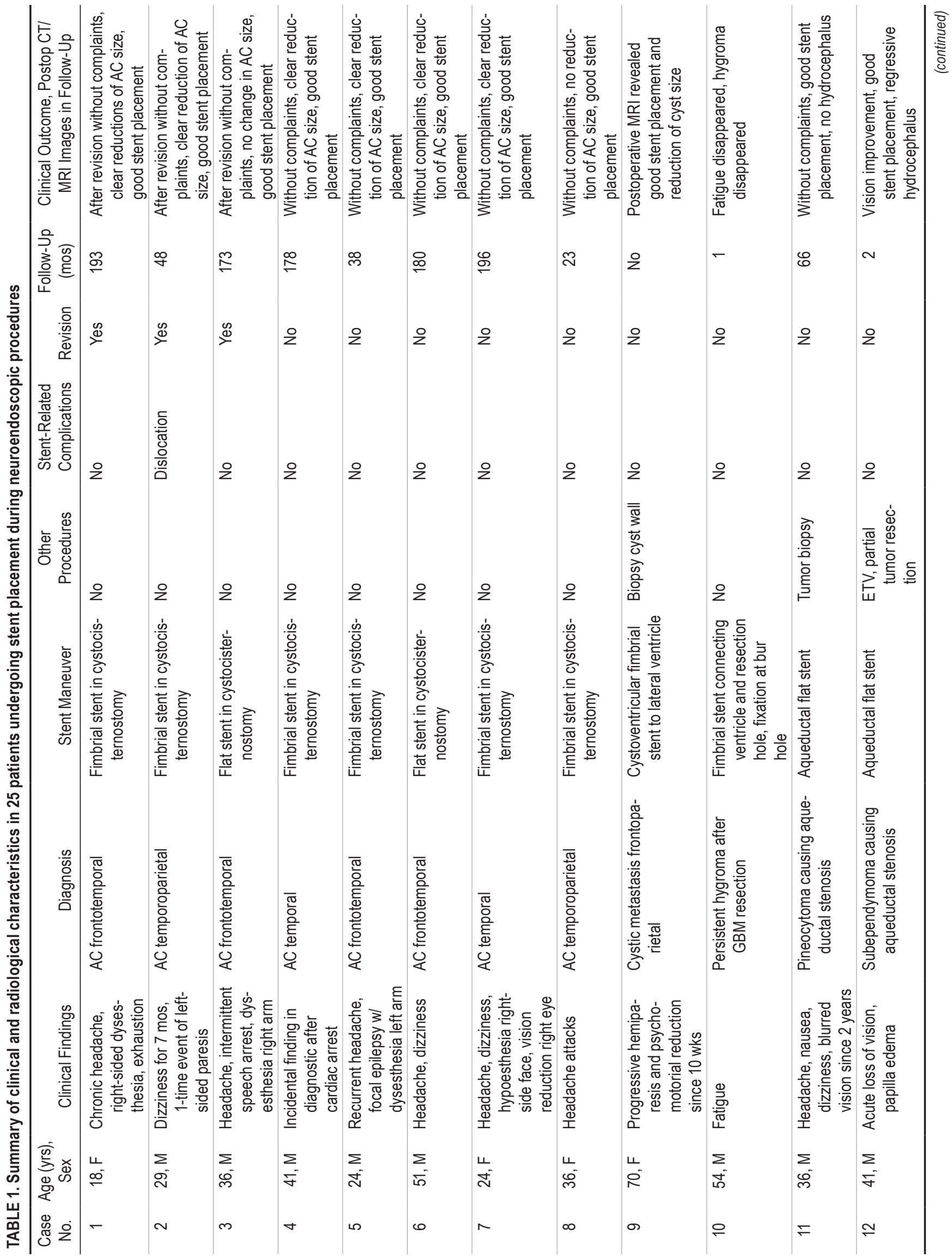




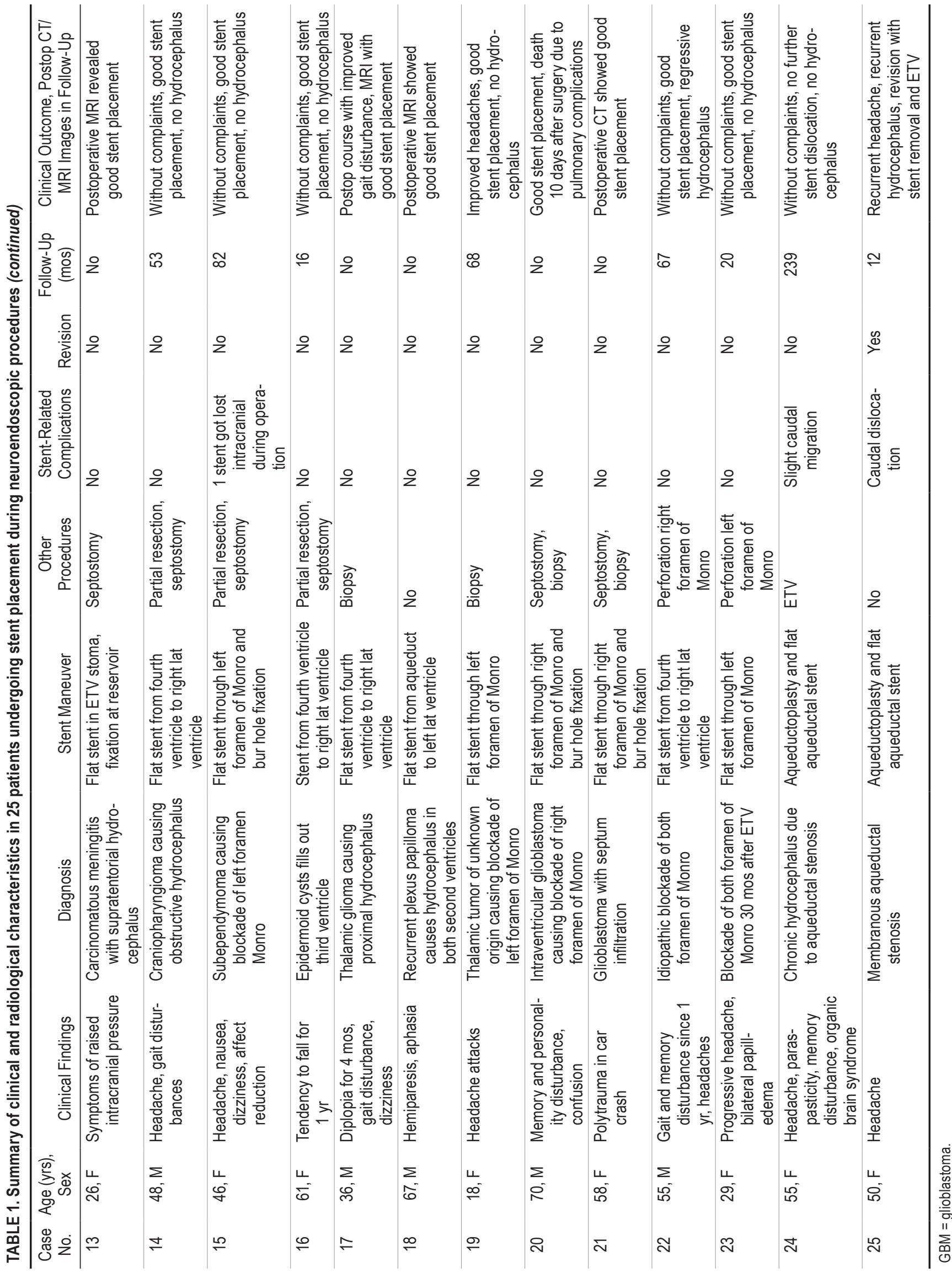




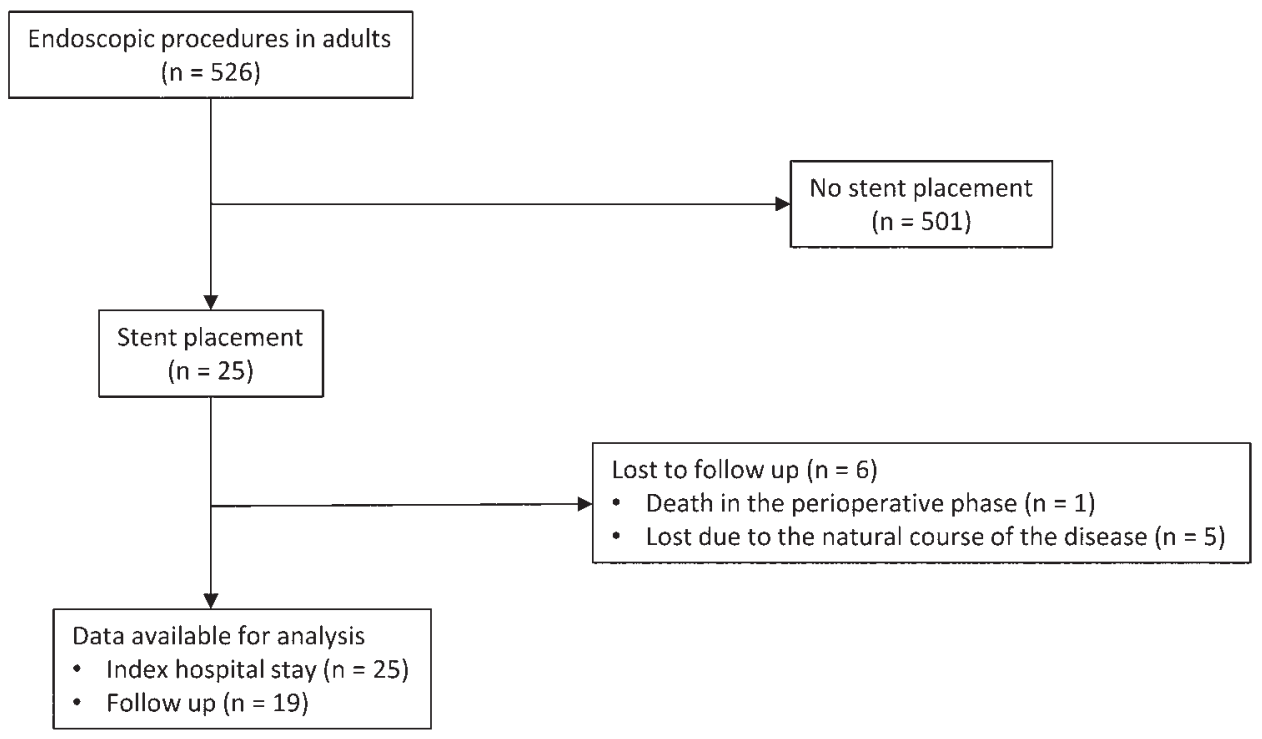

FIG. 1. Flow diagram outlining patient selection. Of 526 endoscopic procedures, 25 were found in which stent placement took place. Follow-up data were available for 19 patients.

crease in cyst size in 6 of 8 cases. Inversion recovery turbo spin-echo sequences are valuable in the postoperative period, because CSF flow can be visualized at the site of the cystocisternostomy and around the stent. This baseline image can then be used for ruling out stent migration or dislocation in the future. Average follow-up for the AC cohort was 129 months (range 23-193 months). Three redo surgeries occurred at 14,24 , and 42 months after surgery in 3 separate patients. One patient presented with dysesthesias and speech arrest in the setting of stent dislocation and acute intracranial hypertension at 42 months (Case 2). Reoperation resulted in immediate reduction of cyst size, reduction of pressure, and resolution of the symptoms. The remaining 2 patients (Cases 1 and 3) suffered from persistent headaches after the initial surgery, and MRI revealed unchanged cyst size. Therefore, elective re-inspection was performed 14 and 24 months after the initial surgeries, respectively. During the second operation, stent patency was apparent, showing communication between the AC and the basal cisterns. Thickened arachnoid membranes behind the stents were loosened and the preexisting cystocisternostomy was enlarged, while the stent was left in place. Follow-up after reoperation for both patients showed improved clinical symptoms. The AC cohort was complicated by the occurrence of 1 transient facial and 1 transient trochlear nerve palsy. Long-term follow-up revealed correct stent position in all patients, reduction of cyst size in 6 , and improvement of clinical symptoms in all treated patients.

\section{Stent Placement for Treatment of Tumor-Related CSF Disorders}

The primary indication for stent placement in the tumor group was threatening obstructive hydrocephalus secondary to tumor growth. In this heterogeneous group, 4 stents were placed just through the foramen of Monro, 4 stents spanned from the fourth ventricle to the lateral ven- tricle through the aqueduct of Sylvius and the foramen of Monro, 2 stents were placed through the aqueduct, and in 1 case the stent was placed in the ETV stoma. In 1 patient, the stent drained a tumor cyst into the lateral ventricle. In another patient, a postoperative hygroma was successfully stented into the ventricular system. Postoperative imaging showed correct stent position in all patients. There was no need for revision surgery in this group, although 5 patients were lost to follow-up. One patient died 2 weeks postoperatively due to respiratory failure. Progressive ventricular dilation and clinical signs of raised intracranial pressure were not observed in any of the treated tumor patients. There were no observed cases of tumor spread attributable to stent placement. These results must be interpreted cautiously given that 5 patients were lost to follow-up, likely due to the natural history of the specific pathologies.

\section{Stent Placement for Treatment of Stenosis of the Foramen of Monro or Aqueductal Stenosis}

One patient presented with de novo occlusion of both foramina of Monro (Case 22). A second patient presented with triventricular hydrocephalus 30 months after a successful ETV (Case 23). In both cases, the hydrocephalus had already created spontaneous septum pellucidum perforations. The stents placed in these 2 patients connected 1 lateral ventricle with the third ventricle, and 1 lateral ventricle with the fourth ventricle, traversing the third, respectively. The clinical condition of these 2 patients improved immediately after stent placement, and ventricular size declined during follow-up.

In 2 patients, aqueductal stenting was performed due to idiopathic stenosis of the aqueduct, causing chronic triventricular hydrocephalus. In 1 patient (Case 24), caudal migration of the aqueductal stent was noted on MRI 1 year after its insertion (Fig. 2). There have been no clinical or radiological signs of raised intracranial pressure to date (239-month follow-up). However, an ETV was performed 


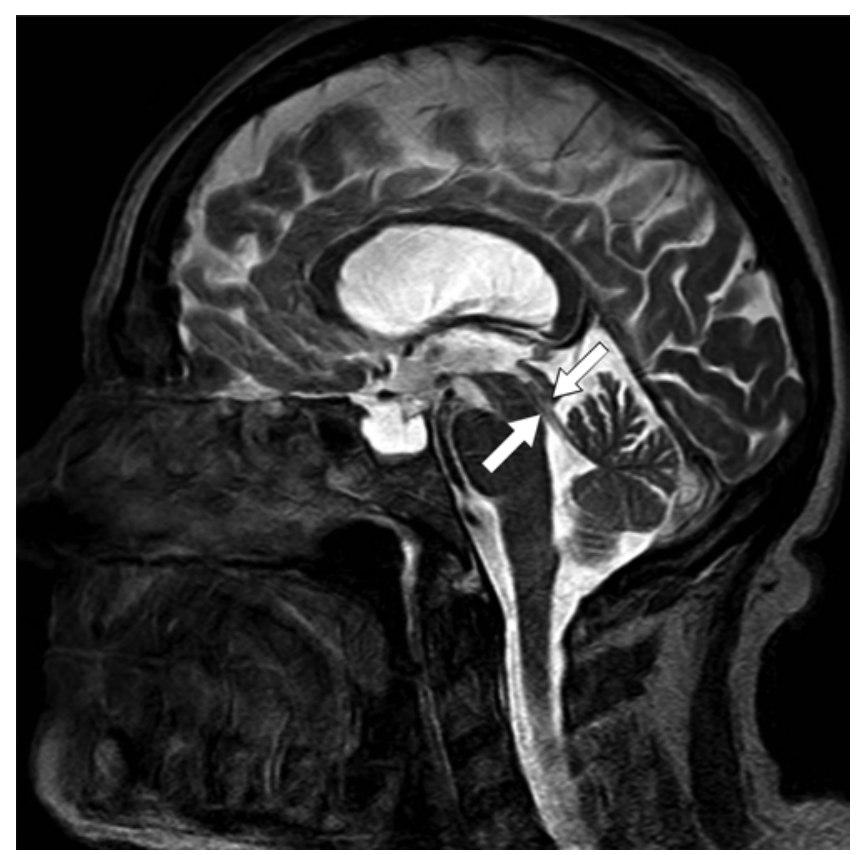

FIG. 2. Sagittal, T2-weighted MRI showing slightly caudal migration of the aqueductal stent (arrows).

at the time of the index operation, and the stoma was still patent with observed flow on sagittal T2 flow studies. In this unique case, it remains unclear whether the ventriculostomy or the aqueductal stent, or both, provided durable CSF flow.

One patient (Case 25) presented with recurrent headaches 12 months after aqueductal stenting. MRI revealed caudal dislocation of the stent, and a successful ETV was performed.

\section{Illustrative Cases \\ Case 10}

A 54-year-old man suffering from a right occipitoparietal glioblastoma underwent resection of recurrent disease 13 months after the initial surgery (Fig. 3A). Reoperation involved opening of the posterior horn of the lateral ventricle that ultimately led to the development of a hemispheric hygroma causing mass effect (Fig. 3B). The patient's primary complaint was unrelenting fatigue. Assuming a valve mechanism between the ventricle and the hygroma, a stent was placed endoscopically, connecting the 2 CSF compartments and fixed in place with a bur hole reservoir. Postoperative imaging demonstrated complete resolution of the hygroma as well as expansion of the ventricle (Fig. $3 \mathrm{C}$ and $\mathrm{D}$ ). This patient was lost to follow-up 4 weeks after surgery.

\section{Case 20}

A 70-year-old man presented with loss of memory and personality disturbances. MRI revealed a giant intraparenchymal and intraventricular tumor compromising the foramen of Monro (Fig. 4A). Neuroendoscopic tumor biopsy was combined with septostomy and placement of a foraminal stent from the right lateral ventricle into the third
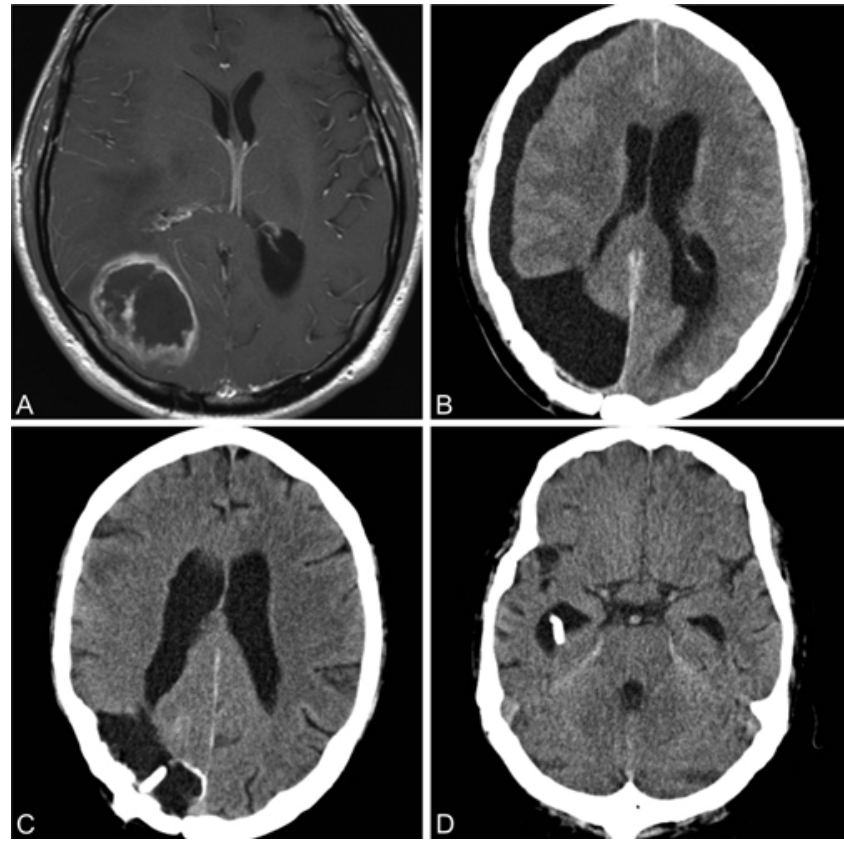

FIG. 3. A preoperative MR image shows a glioblastoma in the rightsided occipitoparietal region (A). After revision, CT revealed a hygroma connected to the resection space and a narrow second ventricle on the right side (B). After stent placement, CT scans show good stent position connecting the right temporal horn with the resection space. The hygroma disappeared and the second ventricle re-enlarged (C and D). All images are axial.

ventricle (Fig. 4B and C). Postoperative CT demonstrated the expected stent position and no further increase in ventricular caliber (Fig. 4D). Histological analysis revealed a glioblastoma. The patient died 2 weeks after surgery due to pulmonary complications.

\section{Case 25}

A 50-year-old woman presented with persistent headache and recurrent triventricular hydrocephalus 4 years after a successful aqueductoplasty for short-segment membranous aqueductal stenosis. A redo aqueductoplasty and stenting of the aqueduct were performed. Postoperative imaging showed correct stent position (Fig. 5 left), and clinical evaluation revealed improved headaches. Twelve months later, her headaches reoccurred in the setting of caudal dislocation of the stent resulting in re-occlusion of the aqueduct with renewed hydrocephalus (Fig. 5 right). Thus, ETV was performed. The stent was left in place and did not cause any problems.

\section{Discussion}

\section{Summary of the Key Results}

The present series establishes the rarity of stent placement during neuroendoscopic procedures in adults. Only $4.8 \%(25 / 526)$ of endoscopic procedures included the placement of a stent. Stenting usually plays an adjunctive role to other endoscopic procedures. The most common indications for stenting include the management of arachnoid cysts and in cases of obstructive hydrocephalus sec- 


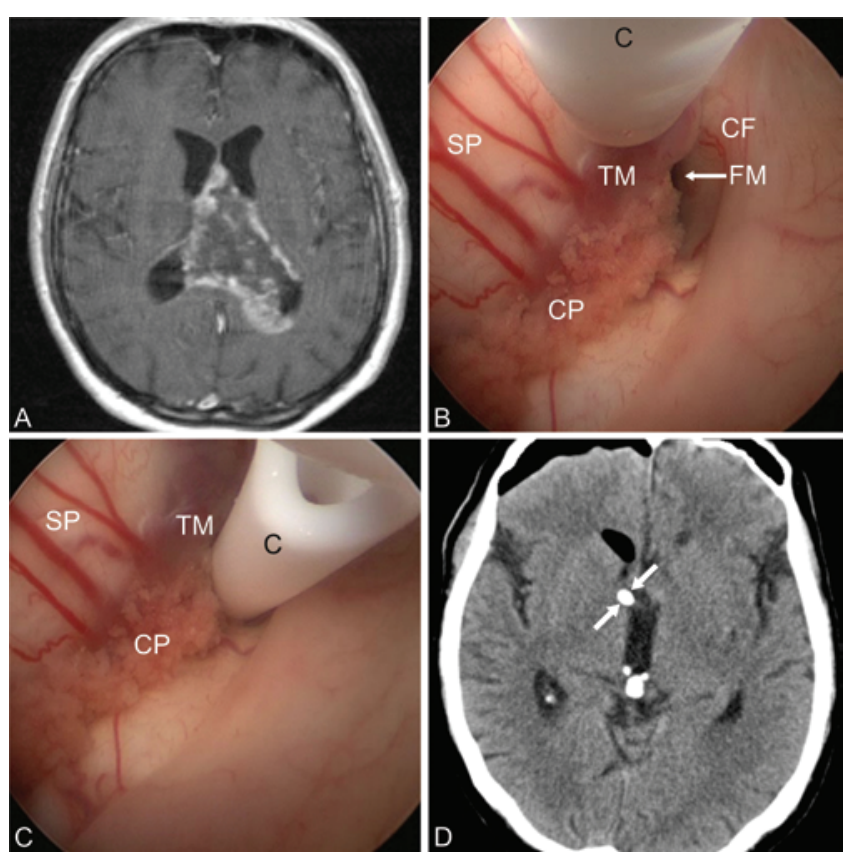

FIG. 4. Preoperative axial MRI shows a giant tumor involving both hemispheres and supratentorial ventricular system (A). Stent placement through the foramen of Monro was performed with the endoscopic technique (B and C). Postoperative axial CT (D) shows regular stent position (arrows). $\mathrm{C}=$ stent; $\mathrm{CF}=$ forniceal columns; $\mathrm{CP}=$ choroid plexus; $\mathrm{FM}=$ foramen of Monro; $\mathrm{SP}=$ septum pellucidum; $\mathrm{TM}=$ tumor.

ondary to tumors. Additionally, cases for stenting of the foramen of Monro or aqueduct due to bi- or triventricular hydrocephalus were recognized. Stent placement was shown to be technically feasible with excellent postoperative clinical results and minimal procedure-related complications. No stent-related infections occurred. Follow-up data revealed stent migration or dislocation in 3 patients $(12 \%)$ with subsequent need for redo surgery in 2 patients (8\%). Seven-year follow-up established the durability of the stenting procedure.

\section{Study Limitations}

Although this study presents favorable results, it is limited by the small number of patients. It is therefore necessary to discuss diagnosis and goals of surgery in detail. Every indication for stent placement was carefully considered with respect to each individual case. The varying pathologies make the number of comparable cases even smaller. Also, the study period spans more than 20 years, a time during which neuroendoscopy has rapidly advanced. For example, stent migration and dislocation have not occurred since stent fixation with a bur hole reservoir has become standard. It is also worth mentioning that the philosophy regarding the management of ACs and idiopathic aqueductal stenosis shifted during this time. All patients in our series, who were treated for an $\mathrm{AC}$ or aqueductal stenosis with stenting, had their procedures performed in the 1990s. Our current practice is to treat ACs with endoscopic cystocisternostomy alone. Patients with idiopathic aqueductal stenosis are treated with ETV alone.

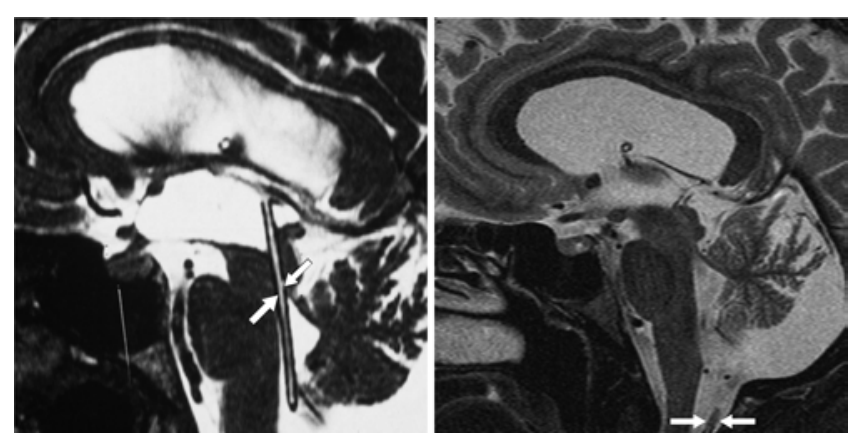

FIG. 5. Postoperative sagittal MRI (left) shows regular stent position (arrows). One year later, the patient presented with recurrent headache. MRI (right) revealed caudal dislocation of the stent (arrows).

Only a few reports exist describing the value and technical feasibility of endoscopic stent placement in adults. ${ }^{8,11,14,24}$ In the following sections, we discuss our results in relation to the literature for each indication for stenting.

\section{Stent Placement Is a Safe Procedure During Neuroendoscopy}

Several authors argue against placement of permanent stents due to possible complications and a lack of longterm results..$^{11,24}$ One complication is stent malposition during the operation. In Case 15 of the present study, 1 stent was lost during the endoscopic procedure and could not be retrieved. The stent, however, did not cause any complications during the follow-up period of 82 months. Stent migration or dislocation can also occur (12\% in our series) but can result in neurological deterioration requiring emergency surgery., ${ }^{3,18}$ In the series of Pitskhelauri et al., 1 of 30 aqueductal stents dislocated into the cerebellar vermis without consequence. ${ }^{17}$ In Cases 2 and 25 of our series, stent dislocation occurred and the patients underwent repeat surgery. In Case 24 of our series, slight downward migration of the stent was noted on 1-year follow-up imaging, but the stent position was stable over the following 19 years. Ersahin et al. observed similar problems, ${ }^{5}$ but no stent migration was reported in other series. ${ }^{1718}$ Rapid change in ventricle size has been reported as a possible association with an increased chance of stent dislodgement. ${ }^{22}$ To avoid stent migration, we advise fixation of the stent to a bur hole reservoir (Fig. 6) that also allows the clinician to tap CSF from the reservoir, if necessary, without the patient who has experienced acute neurological decompensation having to undergo another operation. ${ }^{9,11,16,19}$ To properly anchor the stent, the length of the stent should be measured on the sagittal MRI prior to surgery. ${ }^{22}$ Stent occlusion due to tumor progression and gliosis can also occur. ${ }^{17}$

Although the stent is a foreign body and should have a higher infection risk, in our series, no stent-related infections occurred, which is consistent with multiple other reports. ${ }^{13,17,18}$ In contrast, Ersahin et al. reported the need for stent removal due to ventriculitis in 1 patient. ${ }^{5}$ Patient age appears to be a risk factor for stent-related infections. Thus, in children $<1$ year of age, antibiotic-labeled catheters may be used. ${ }^{22}$ 


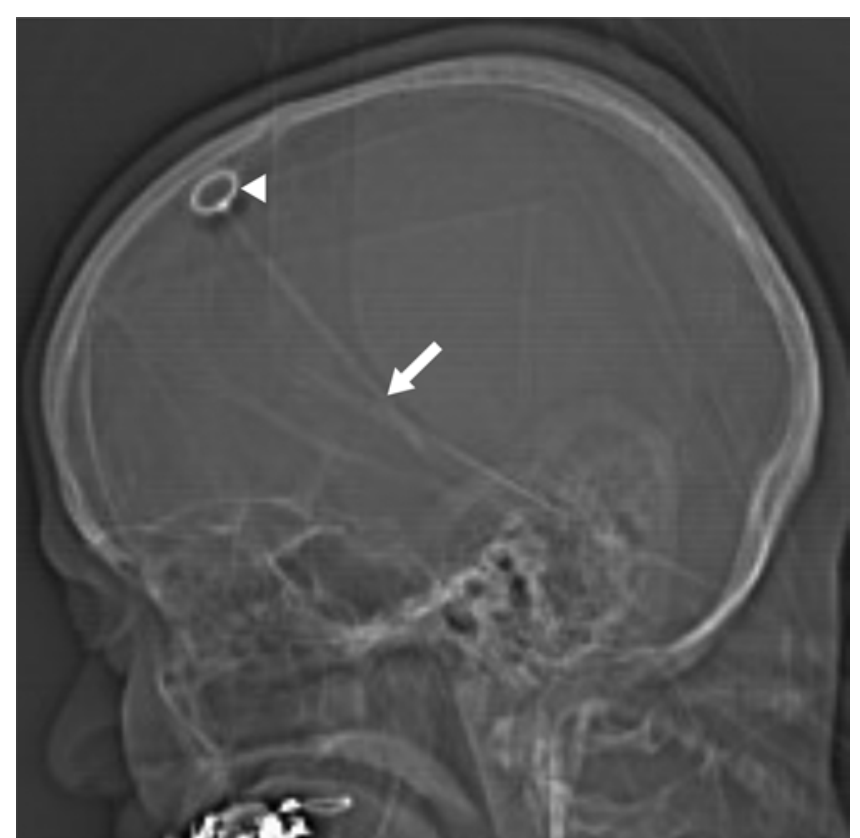

FIG. 6. Postoperative lateral radiograph of the head (Case 21) shows a stent (arrow) connecting the lateral and third ventricles with stent fixation at the bur hole (triangle).

\section{Stent Placement for Treatment of Tumor-Related CSF Disorders}

Intraventricular tumors are an ideal indication for neuroendoscopy. In 1978, Fukushima described a tumor biopsy using the endoscopic technique. ${ }^{10,14}$ Tumor biopsy and partial tumor resection can be combined with CSF diversion through a single approach, potentially resulting in a shorter hospital stay. ${ }^{12,14,19,23}$ An additional advantage of stent placement in tumor patients is the reduction in shuntrelated complications, including the risk of intraperitoneal spread of tumor cells. ${ }^{17}$ Hydrocephalus with aqueductal stenosis due to tumor involvement can be treated by ETV or aqueductal stenting in select patients. ${ }^{6,8,9,14,19} \mathrm{In}$ a series by Pitskhelauri et al. in 84 patients, tumor resection and restoration of the CSF pathway was performed during 1 procedure. Fifty-five ETVs and 30 aqueductal stent placements were performed with the microsurgical technique. The success rate of aqueductal stent placement was higher than that of ETV in this series. However, Pitskhelauri performed the stent procedures with the microsurgical technique. ${ }^{17}$ In a series of Berlis et al., tumor cysts were stented into the ventricle by using stereotactic planning and the endoscopic technique. ${ }^{1}$ In the present study, all procedures to restore CSF flow or prevent tumor-induced CSF blockade demonstrated good clinical and radiological results despite the limited follow-up proscribed by the natural history of the primary diseases.

\section{Stent Placement for Treatment of ACs}

In the series of Fulkerson et al., 3 stents were placed in 79 cases of posterior fossa ACs. ${ }^{11}$ All 3 cases showed good results. Our interventions in the 1990s more frequently included placement of a stent through the cystocisternostomy at the time of the index surgery. Today, we use stent placement in ACs only as salvage treatment for redo operations or in cases with restricted anatomical conditions limiting the size of the cystocisternostomies. In our patients with ACs, a radiological decrease in cyst size was noted in $75 \%$ of patients and clinical improvement was observed in all. Clinical and radiological outcomes do not necessarily correlate in the surgical treatment of AC..$^{11,13}$

\section{Stent Placement for Treatment of Stenosis of Foramen of Monro or Aqueductal Stenosis}

Very few reports exist on neuroendoscopic stent placement for bilateral occlusion of the foramen of Monro. ${ }^{15}$ Thus, Case 23 of our series has been published as a case report previously. ${ }^{4}$ The 2 patients with stenting of the foramen of Monro from this series (Cases 22 and 23) demonstrated good clinical and radiological results in the follow-up period. Because our observation suggests a risk of re-occlusion after foraminoplasty, stent placement is a reliable alternative to implantation of a permanent shunt in this rare clinical entity. In Case 22, we placed a stent connecting the fourth ventricle with the lateral ventricle. This was performed to get a longer stent to reduce the risk of stent migration. In our most recent case (Case 23), we just placed a stent from the third to the lateral ventricle. In our current practice, we would recommend stabilizing the stent by bur hole fixation and using a shorter stent. Both cases of aqueductal stenting (Cases 24 and 25) were complicated by stent migration or dislocation. If the stents had been fixed to a bur hole reservoir, as is our practice today, we believe these complications would have been avoided. However in our current practice, we advise ETV instead of aqueductal stenting in these situations.

\section{Generalizability}

The data from this series allow us to provide some general advice for the use of neuroendoscopic stent placement in adults. First, infection risk is minimal with stenting procedures and should not be a significant factor when weighing the risks and benefits of the procedure. Second, stent use, independent of indication, should always be secured at the bur hole site to prevent dislocation or dislodgement. Third, stenting should be considered a salvage procedure for aqueductal stenosis, foraminal stenosis, and ACs, but may be considered as the primary intervention for tumorrelated impediment to CSF flow.

\section{Conclusions}

Stent placement during neuroendoscopic procedures in adults is a rare event (4.8\%). It may be indicated when reliable and long-lasting restoration of CSF pathway obstructions cannot be achieved with standard endoscopic techniques, such as ETVs, fenestrations, foraminoplasties, septostomies, or aqueductoplasties. In the treatment of tumor-related hydrocephalus, it is a good option to avoid reclosure of the restored CSF pathway by tumor growth that finally may result in external drainage or shunting, not only in palliative situations. At this time, we do not recommend routine stent placement after endoscopic fenestration of ACs or for idiopathic aqueductal stenosis. To avoid 
stent migration or dislocation, the stent should be fixed to a bur hole reservoir.

\section{Acknowledgments}

Michael R. Gaab, MD, PhD, is the former head of the Department of Neurosurgery, Hannover Nordstadt hospital.

\section{References}

1. Berlis A, Vesper J, Ostertag C: Stent placement for intracranial cysts by combined stereotactic/endoscopic surgery. Neurosurgery 59 (4 Suppl 2):ONS474-ONS479, 2006

2. Cinalli G, Spennato P, Savarese L, Ruggiero C, Aliberti F, Cuomo L, et al: Endoscopic aqueductoplasty and placement of a stent in the cerebral aqueduct in the management of isolated fourth ventricle in children. J Neurosurg 104 (1 Suppl):21-27, 2006

3. da Silva LR, Cavalheiro S, Zymberg ST: Endoscopic aqueductoplasty in the treatment of aqueductal stenosis. Childs Nerv Syst 23:1263-1268, 2007

4. El Refaee E, Baldauf J, Schroeder HW: Bilateral occlusion of the foramina of Monro after third ventriculostomy. J Neurosurg 116:1333-1336, 2012

5. Erşahin Y: Endoscopic aqueductoplasty. Childs Nerv Syst 23:143-150, 2007

6. Erşahin Y, Kesikçi H: Endoscopic management of quadrigeminal arachnoid cysts. Childs Nerv Syst 25:569-576, 2009

7. Esposito F, Di Rocco F, Zada G, Cinalli G, Schroeder HW, Mallucci C, et al: Intraventricular and skull base neuroendoscopy in 2012: a global survey of usage patterns and the role of intraoperative neuronavigation. World Neurosurg 80:709-716, 2013

8. Fritsch MJ, Kienke S, Mehdorn HM: Endoscopic aqueductoplasty: stent or not to stent? Childs Nerv Syst 20:137-142, 2004

9. Fritsch MJ, Schroeder HW: Endoscopic aqueductoplasty and stenting. World Neurosurg 79:S20.e15-S20.e18

10. Fukushima T: Endoscopic biopsy of intraventricular tumors with the use of a ventriculofiberscope. Neurosurgery 2:110113,1978

11. Fulkerson DH, Vogel TD, Baker AA, Patel NB, Ackerman LL, Smith JL, et al: Cyst-ventricle stent as primary or salvage treatment for posterior fossa arachnoid cysts. J Neurosurg Pediatr 7:549-556, 2011

12. Gaab MR, Schroeder HW: Neuroendoscopic approach to intraventricular lesions. J Neurosurg 88:496-505, 1998

13. Gallo P, Szathmari A, Simon E, Ricci-Franchi AC, Rousselle C, Hermier M, et al: The endoscopic trans-fourth ventricle aqueductoplasty and stent placement for the treatment of trapped fourth ventricle: long-term results in a series of 18 consecutive patients. Neurol India 60:271-277, 2012

14. Mohanty A, Santosh V, Devi BI, Satish S, Biswas A: Efficacy of simultaneous single-trajectory endoscopic tumor biopsy and endoscopic cerebrospinal fluid diversion procedures in intra- and paraventricular tumors. Neurosurg Focus 30(4):E4, 2011
15. Mori H, Koike T, Fujimoto T, Nishiyama K, Yoshimura J, Tanaka R: Endoscopic stent placement for treatment of secondary bilateral occlusion of the Monro foramina following endoscopic third ventriculostomy in a patient with aqueductal stenosis. Case report. J Neurosurg 107:416-420, 2007

16. Oertel JM, Baldauf J, Schroeder HW, Gaab MR: Endoscopic options in children: experience with 134 procedures. J Neurosurg Pediatr 3:81-89, 2009

17. Pitskhelauri DI, Konovalov AN, Kornienko VN, Serova NK, Arutiunov NV, Kopachev DN: Intraoperative direct third ventriculostomy and aqueductal stenting in deep-seated midline brain tumor surgery. Neurosurgery 64:256-267, 2009

18. Sagan LM, Kojder I, Poncyljusz W: Endoscopic aqueductal stent placement for the treatment of a trapped fourth ventricle. J Neurosurg 105 (4 Suppl):275-280, 2006

19. Schroeder HW: Intraventricular tumors. World Neurosurg 79 (2 Suppl):S17.e15-S17.e19, 2013

20. Schroeder HW: A new multipurpose ventriculoscope. Neurosurgery 62:489-492, 2008

21. Schroeder HW, Oertel J, Gaab MR: Endoscopic treatment of cerebrospinal fluid pathway obstructions. Neurosurgery 62 (6 Suppl 3):1084-1092, 2008

22. Schulz M, Goelz L, Spors B, Haberl H, Thomale UW: Endoscopic treatment of isolated fourth ventricle: clinical and radiological outcome. Neurosurgery 70:847-859, 2012

23. Teo C, Kadrian D, Hayhurst C: Endoscopic management of complex hydrocephalus. World Neurosurg 79 (2 Suppl):S21. e1-S21.e7, 2013

24. Tirakotai W, Riegel T, Schulte DM, Bertalanffy H, Hellwig D: Neuroendoscopic stent procedure in obstructive hydrocephalus due to both foramina of Monro occluding craniopharyngioma: technical note. Surg Neurol 61:293-296, 2004

\section{Disclosures}

Henry W. S. Schroeder and Michael R. Gaab are consultants for Karl Storz GmbH \& Co. KG, Tuttlingen, Germany.

\section{Author Contributions}

Conception and design: Marx, Schroeder, Baldauf. Acquisition of data: Marx, Fleck, El Refaee, Vorbau. Analysis and interpretation of data: Marx, Fleck, El Refaee, Manwaring, Fritsch, Gaab, Schroeder, Baldauf. Drafting the article: Marx, Schroeder, Baldauf. Critically revising the article: Marx, Fleck, El Refaee, Manwaring, Fritsch, Gaab, Schroeder, Baldauf. Reviewed submitted version of manuscript: Marx, Vorbau. Approved the final version of the manuscript on behalf of all authors: Marx. Statistical analysis: Marx, Baldauf. Administrative/technical/material support: Marx. Study supervision: Marx, Schroeder, Baldauf.

\section{Correspondence}

Sascha Marx, Department of Neurosurgery, University of Greifswald, Sauerbruchstraße, 17475 Greifswald, Germany. email: marxs@uni-greifswald.de. 